\title{
Estimating extreme flood events - assumptions, uncertainty and error
}

\author{
S. W. Franks ${ }^{1}$, C. J. White ${ }^{1}$, and M. Gensen ${ }^{2}$ \\ ${ }^{1}$ School of Engineering and ICT, University of Tasmania, Hobart, Australia \\ ${ }^{2}$ University of Twente, Faculty of Engineering Technology, Enschede, the Netherlands
}

Correspondence to: S. W. Franks (stewart.franks@utas.edu.au)

Received: 9 April 2015 - Accepted: 9 April 2015 - Published: 11 June 2015

\begin{abstract}
Hydrological extremes are amongst the most devastating forms of natural disasters both in terms of lives lost and socio-economic impacts. There is consequently an imperative to robustly estimate the frequency and magnitude of hydrological extremes. Traditionally, engineers have employed purely statistical approaches to the estimation of flood risk. For example, for an observed hydrological timeseries, each annual maximum flood is extracted and a frequency distribution is fit to these data. The fitted distribution is then extrapolated to provide an estimate of the required design risk (i.e. the $1 \%$ Annual Exceedance Probability - AEP). Such traditional approaches are overly simplistic in that risk is implicitly assumed to be static, in other words, that climatological processes are assumed to be randomly distributed in time. In this study, flood risk estimates are evaluated with regards to traditional statistical approaches as well as Pacific Decadal Oscillation (PDO)/El NiñoSouthern Oscillation (ENSO) conditional estimates for a flood-prone catchment in eastern Australia. A paleoreconstruction of pre-instrumental PDO/ENSO occurrence is then employed to estimate uncertainty associated with the estimation of the $1 \%$ AEP flood. The results indicate a significant underestimation of the uncertainty associated with extreme flood events when employing the traditional engineering estimates.
\end{abstract}

\section{Introduction}

The use of empirical flood frequency analysis is widespread throughout hydrological practice - however flood risk estimation is typically achieved through relatively simple statistical analyses of relatively short data series without any regard to the climatological conditions that produce floods. The basic assumption underlying empirical flood frequency analysis is that annual maximum flood peaks are distributed independently and identically (iid). The implied assumption is that the climate is statistically 'static' at all timescales and the risk of a flood of a given magnitude is taken as being the same from one year to the next, irrespective of the underlying climate mechanisms. Whilst the iid assumption may hold in many locations, if violated, this may lead to substantially biased estimates of both short- and long-term risk.

Additionally, in recent decades there has been concern over anthropogenically-induced climate change - namely that human inputs of carbon dioxide and other radiatively active gases will, or are already have, change the radiative energy balance of the planet. Such a change may lead to irrevocable climate change. Over more recent years, it has been suggested that regional climates will be marked by an enhanced hydrological cycle resulting in substantial changes to flood frequency and severity. Such concerns over possible anthropogenic climate change has resulted in numerous studies that have sought to employ statistical analyses of trends in hydrologic timeseries in the hope of identifying a consistent climate change "signal" (e.g. Kundzewicz and Robson, 2004). Typically these studies utilise simple statistical tests for trend and/or step changes in observed flood sequences. The key to climate change detection approaches is that if a trend is identified and cannot be attributed to other changes, for instance land use changes, then anthropogenic climate change is the most likely cause. A possible problem with these approaches, however, is the implicit assumption of hydrologic stationarity. In common with typical engineering approaches to flood risk estimation, hydrologic time series are assessed statistically without any understanding of the under- 


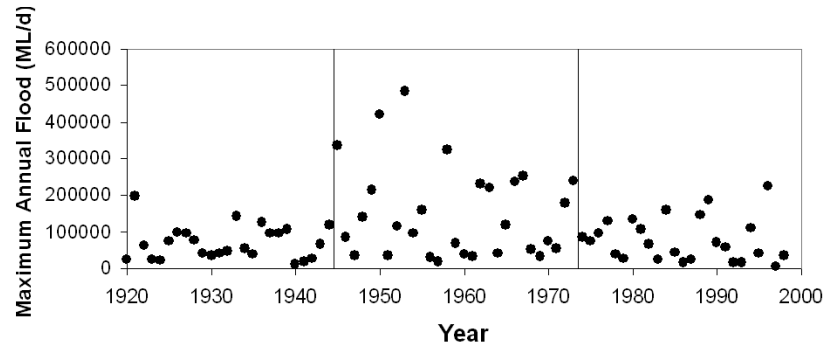

Figure 1. Annual maximum timeseries for a northern NSW catchment. IPO/PDO phase changes indicated by vertical lines.

lying climate processes that produce hydrologic variability for entirely natural reasons.

In this study, the empirical evidence for natural long-term changes in flood frequency is examined for eastern Australia. In particular, the causal climate mechanisms for changes in flood regimes are investigated using known documented climatological phenomena such as the El Niño-Southern Oscillation (ENSO). Subsequently, the assumption of stationarity of flood risk is explored with regard to the traditional engineering estimation of flood risk. The role of non-stationary flood risk in confounding climate change detection methodologies is also explored.

\section{Empirical studies of variability and change in eastern Australia}

It is well known that Australia experiences one of the most markedly variable climates; numerous studies have previously documented regional climate shifts across Australia. Importantly, there is an abundance of evidence that climate variables affecting Australia shifted significantly during the 1940s. In particular, Cornish (1977) noted a marked and abrupt change in annual average rainfall across eastern Australia occurring around 1945. Related to these observations of rainfall change, Allan et al. (1995) showed that Indian Ocean Sea Surface Temperatures (SST) were cooler at mid-latitudes and warmer in the subtropical latitudes in the periods 1900 to 1941 when compared with the period 1942 to 1983 . In addition they found similar anomalies in surface winds, concluding that the "semi-permanent anticyclone in the mean flow field of the atmosphere over the southern Indian Ocean in the austral summer was weaker in the first 42 years of the 1900s".

In parallel with these studies noting change in standard meteorological rainfall, Erskine and Warner (1988) investigating floods, sediments and geomorphological changes in eastern Australia identified what they termed Flood- and Drought- Dominated Regimes (FDR/DDR). The essence of the concept is that eastern Australia regularly experiences shifts from one climatological state to another, whereby high rainfall periods lead to marked variability in sediment deliv-

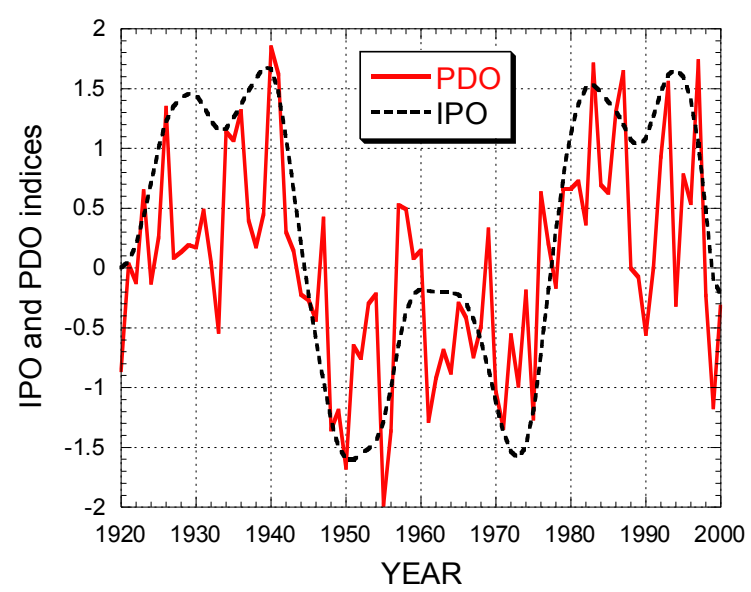

Figure 2. IPO/PDO indices.

ery and accumulation. In a study of instrumental annual maximum flood series, Franks and Kuczera (2002) also demonstrated there is an apparent shift in flood frequency across eastern Australia. Utilising 41 gauges located across New South Wales (NSW), 37 of these (or 90\%) could be show to have experienced an increase in flood risk after 1945. Franks (2002) demonstrated that the change in the instrumental flood frequency record could be objectively identified as being broadly in line with the previous observations of a shift in annual rainfall and circulation patterns as noted above.

To demonstrate the marked shifts in eastern Australian flood risk, Fig. 1 shows a typical annual maximum flood series from a representative flow gauge from the region. As can be seen, a marked increase in flood risk is apparent around 1945. Of interest, the mean annual maximum flood post-1945 is approximately twice that occurring in the pre-1945 period. Also apparent in Fig. 1 is a return to lower flood risk occurring around the mid-1970's. Figure 1 demonstrates that the assumption that individual annual maximum flood events are clustered and therefore do not satisfy the iid assumption. This record and the majority of others across eastern Australia indicate that some process or processes must be operating that dictate structure in terms of temporal patterns in the instrumental flood records.

It is clear from the instrument records both of rainfall and floods, as well as from sedimentary records, that eastern Australia has experienced marked changes in flood risk over decadal timescales. Climatological processes, such as ENSO and Interdecadal Pacific Oscillation (IPO)-PDO, appear to have played a strong role in determining such variability. Figure 2 demonstrates the change in IPO-PDO between 1945 and 1975. This change was associated with an increased frequency of flood-bearing La Niña events (Kiem et al., 2003). There are a number of potential implications arising from these observations: 


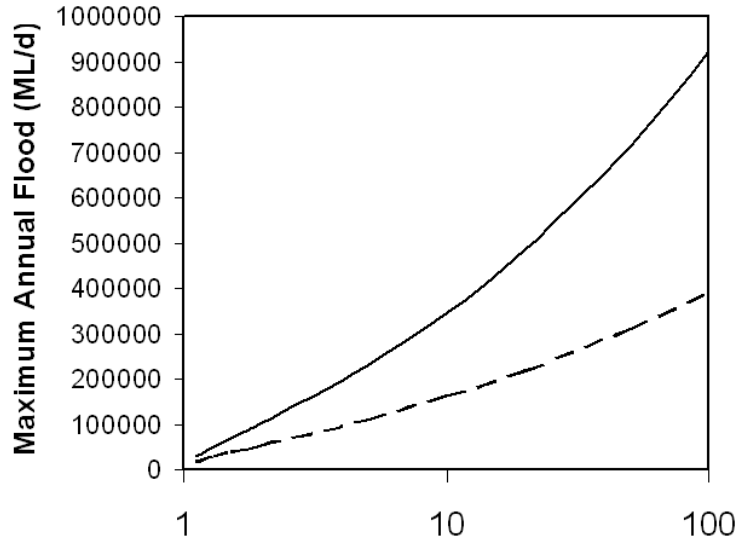

Average Return Interval (yrs)

Figure 3. Flood frequency curves for IPO/PDO negative (solid line) and IPO/PDO positive (dashed line).

- traditional flood frequency based on simple statistics and limited data may be erroneous if no account of the prevailing climate state is included in flood risk estimation

- studies seeking to attribute changes in flood frequency as evidence of anthropogenic climate change may reveal trends that are largely natural in origin

The following sections seek to quantify the possible error and uncertainty arising both in flood risk estimation and in climate change attribution

\section{Assessing uncertainty in long-term flood risk estimation arising from limited samples of multi-decadal variability}

To assess the uncertainty associated with the iid assumption employed in traditional flood risk estimation, a simple Monte Carlo based methodology is developed. The representative series of annual maximum flood data shown earlier in Fig. 1 was employed. These data were stratified according to the positive and negative phases of the IPO-PDO. Log-normal flood frequency distributions associated with each of these phases were calculated. Figure 3 shows marked differences in the resultant flood frequency curves with the IPO negative phase. This figure demonstrates a statistically significant increase of a factor of 2.5 in the $1: 100$ year flood risk, an increase typical across eastern Australia (Micevski et al., 2006).

To generate feasible Monte Carlo realisations of long-term flood risk, a pre-instrumental PDO reconstruction is employed. This series of IPO-PDO phase transitions was derived from assessing multiple proxy reconstructions for Pacific climate variability, whereby statistically significant step

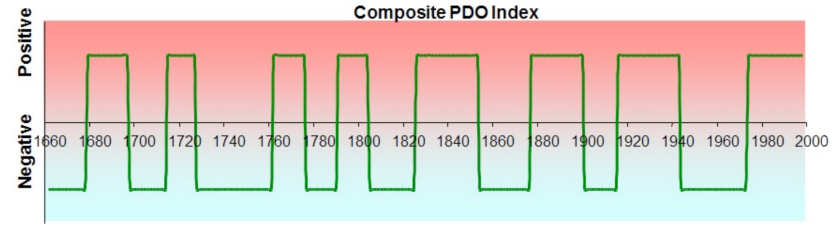

Figure 4. Composite PDO reconstruction from multiple paleo records.

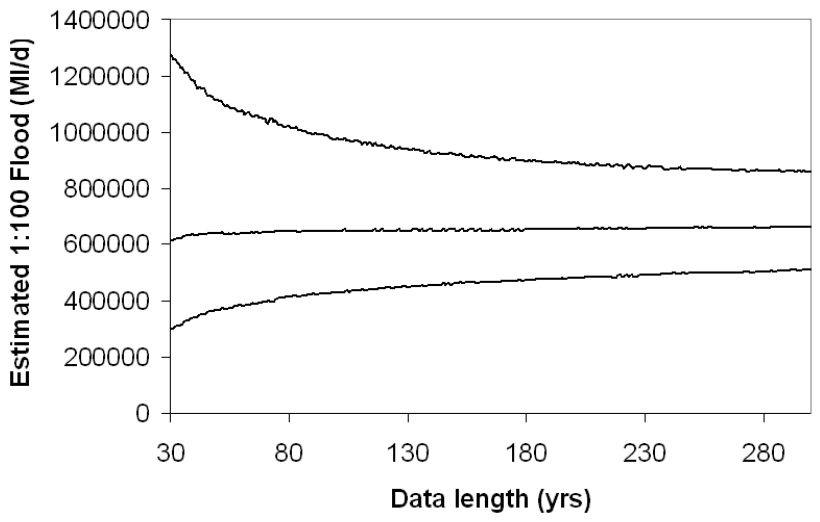

Figure 5. Median and $90 \%$ uncertainty quantiles of estimated $1 \%$ AEP from randomly sampled Monte Carlo replicates.

changes in the derived timeseries where evaluated for coherence. A composite IPO-PDO index of phase transitions was then derived (Fig. 4); see Verdon and Franks (2006) for more details. 10000 individual sub-samples of the composite IPO-PDO timeseries were then randomly generated of length $n$ years. Monte Carlo generated annual maximum flood series were then sampled from the flood frequency distributions associated with the corresponding IPO-PDO phase. The resultant distribution of the $1: 100$ year flood were then calculated. This process was repeated for values of $n$ from 30 to 300 years.

To demonstrate the uncertainty of the mean annual flood risk as a function of available data length, $n$, and unknown IPO-PDO climate state, Fig. 5 shows the simulated median and $90 \%$ uncertainty envelope of the Monte Carlo derived distribution corresponding to the uncertainty envelope for the estimated 1:100 year flood, a common criterion in practical engineering and planning processes. As can be seen, the uncertainty envelope is particularly wide for data lengths of 30 years, indicating a $10 \%$ chance of being over a factor of 3 in error. Whilst Fig. 5 does indicate a reduction in the upper and lower uncertainty limits as the available data length, $n$, increases it is worthwhile to note that even with 150 years of data the residual uncertainty remains very large indicating a $10 \%$ chance of being in error by over a factor of 2 . 

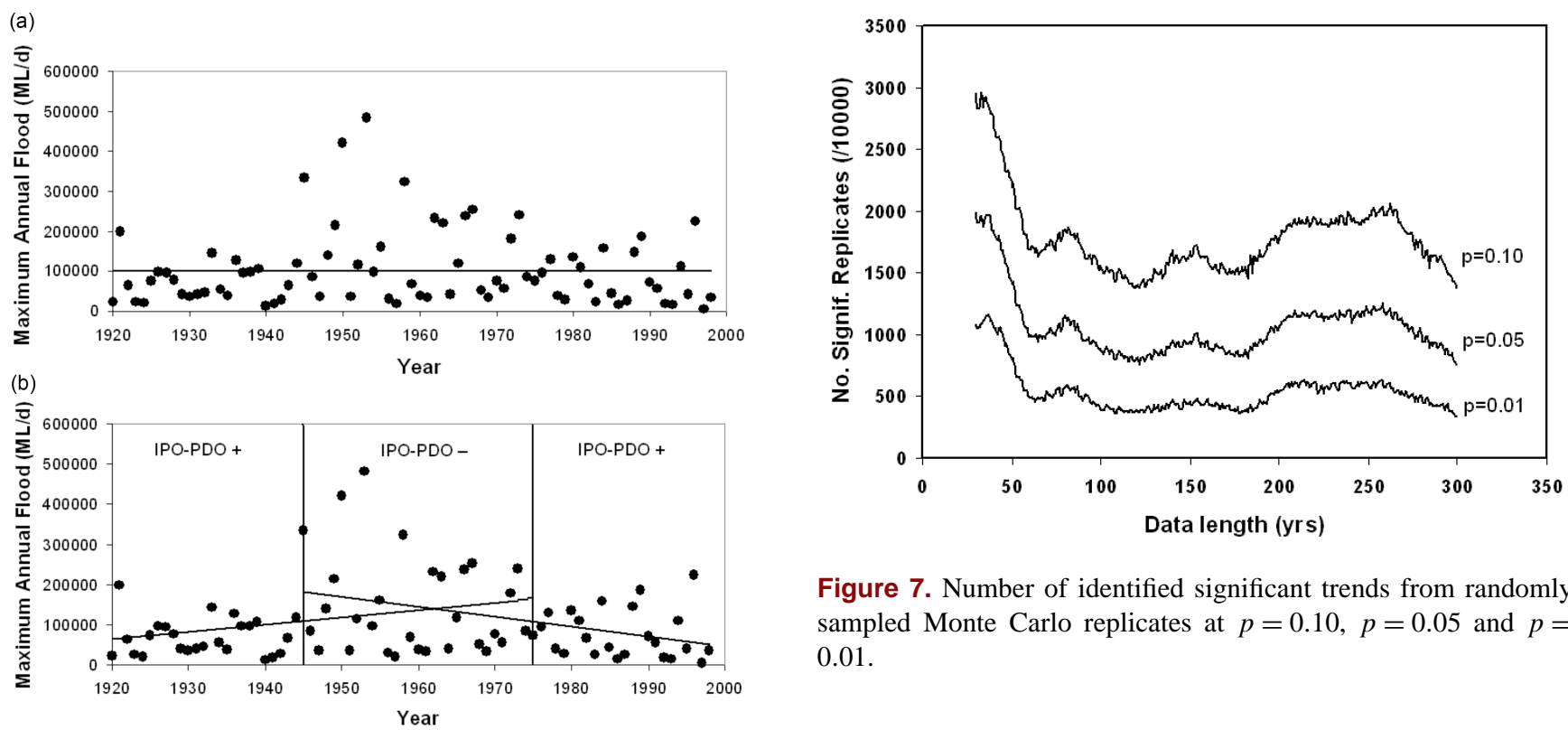

Figure 7. Number of identified significant trends from randomly sampled Monte Carlo replicates at $p=0.10, p=0.05$ and $p=$ 0.01 .

Figure 6. Trends in annual maximum flood timeseries for all years. (b) Trends in annual maximum flood timeseries for 1920-1975 and 1945-1998.

\section{Implications for empirical climate change detection methodologies}

As noted earlier, the current concern over anthropogenicallyinduced climate change has led to a number of studies whereby trends and changes are assessed in hydrological records with the aim of climate change attribution. Perhaps the simplest form of trend detection is a simple linear trend applied to an available timeseries. To assess the potential for significant error in such methodologies (or misattribution), Fig. $6 \mathrm{a}$ and $\mathrm{b}$ show the employed representative timeseries with (a) a linear trend applied to all years (1920-2000), and (b) linear trends applied from 1920-1975 and 1945-1998. Figure 6a shows no discernable trend when all available data are available (gradient $=-21 \mathrm{Ml} \mathrm{d}^{-1} \mathrm{yr}^{-1} ; p=0.963$ ). If we were back in 1975, as shown in Fig. 6b, a marked upward trend in flood risk (gradient $=1837 \mathrm{Ml} \mathrm{d}^{-1} \mathrm{yr}^{-1}$; $p=0.0528$ ) would have been identified. However, if records only began in 1945, then a marked and significant downward trend in flood risk (gradient $=-2471 \mathrm{Ml} \mathrm{d}^{-1} \mathrm{yr}^{-1}$; $p=0.0188$ ) would have been found. In the presence of multi-decadal variability, the length of sample available as well as the sampling of the prevailing multi-decadal climate states could lead to inaccurate, but statistically significant, trends.

More typically, a Mann-Kendall non-parametric test for monotonic trends is employed in the identification of change in hydrological flood timeseries (see Kundzewicz and Robson, 2004, for an overview of alternative methodologies). Interestingly, when applied to the two sub-series, the identi-

fied trends become even more highly statistically significant (1920-1975: $p=0.017$; 1945-1998: $p=0.008)$. Such high levels of statistical confidence are incorrect in the presence of multi-decadal variability. Put simply, the assumptions about the data employed in the tests are invalid.

To assess the possible errors associated with identifying statistically significant trends associated with the presence of multi-decadal variability in flood series, the Monte Carlo-derived sequences derived above were each individually tested for monotonic trends using the Mann-Kendall test. Each occurrence of significance was recorded at the 10,5 and $1 \%$ significance levels. The fraction of Monte Carlo replicates corresponding to significant trends was then plotted as a function of sample length, $n$. Figure 7 shows the number of replicates out of 10000 that returned significant trends as a function of multi-decadal flood variability and sample data length. Figure 7 also shows that utilizing 30 years of data, almost $30 \%$ of replicates are found to contain significant trends at the $10 \%$ level, $20 \%$ at the $5 \%$ level and $15 \%$ at the $1 \%$ level. These represent marked error in the application of the test in the presence of multi-decadal variability. It should also be noted that as the length of data in a sample increases, the fraction of errors decrease but do not disappear even after 300 years.

As noted by Kundzewicz et al. (2004), hydrological timeseries that display marked variability may be effectively filtered through the analysis of serial correlation in the records, however an open question is how should one arbitrarily select a threshold significance level of serial correlation on which to accept or reject individual timeseries? Moreover, it is entirely feasible that individual regional climate may display only periodic epochs of elevated/reduced flood risk as a complex function of the interaction of climate modes, unlike the periodic timeseries employed here. This would further confound attempts to filter datasets prior to trend analysis. Ulti- 
mately, the application of simple statistical techniques cannot provide a definitive analysis of the causes of changes in hydrological timeseries without robust understanding of the causal mechanisms that lead to floods.

\section{Conclusions}

Flood risk estimation is a cornerstone of practical engineering and planning processes. The consequences of significant error in flood risk estimation techniques are substantial. In this study, it has been demonstrated that traditional flood risk estimation techniques based on simple statistical analysis of relatively short flood histories may result in large biases when applied to catchments that experience marked multidecadal climate variability. Using the traditional iid assumptions, 30 years of data are typically deemed an adequate minimum for robust flood risk estimation. The results presented here demonstrate that uncertainty in both the mean annual maximum and the $1: 100$ year flood risk may be in error by as much as a factor of 3 when only limited data are available. Whilst this uncertainty reduces as a function of available data length, the residual uncertainty still remains high when compared to the invalid uncertainty estimates under the iid assumption. These results indicate the importance of process understanding in providing appropriate flood risk estimates given both multi-decadal variability and limited instrumental records.

The approach adopted contains a number of specific assumptions. Above all, the approach adopted assumes that whilst flood risk varies on multi-decadal timescales according to the IPO-PDO, it also assumes that this association is itself stable. In other words, that the association between IPOPDO and variable flood risk observed within the instrumental record (typically the twentieth century) holds for the centuries prior. It is entirely possible that at longer timescales than the instrumental record other processes may mean a more or less variable association between the IPO-PDO and eastern Australian flood risk. From the viewpoint of the requirement to provide robust flood risk estimates and their uncertainty that may need to be equally applicable now as in 100 years time, the assumption seems suitably conservative. This assumption can be tested through the development of appropriate and robust proxy measures of pre-instrumental climatic risk in eastern Australia. This is the subject of current research and will be reported in the future.

An additional assumption is that anthropogenic effects on climate have been negligible over the period of the instrumental record. As noted by Kundzewicz and Robson (2004), analyses of long-term flood risk from over 195 quality gauged stations has not provided any clear indication as to how anthropogenic climate change may or may not affect flood risk. It remains possible that anthropogenic emissions of radiatively active gases may affect flood extremes, however it is clear that historic variability on multi-decadal timescales has been a destructive feature of natural Australian climate variability prior to recent concerns over climate change. On the basis of the available evidence it appears that it should remain the primary concern for the immediate future.

Whilst this paper has focused on eastern Australian flood data, it should also be appreciated that ENSO is a quasiglobal phenomena, affecting regional climates across the world. Significant impacts have been detected across Asia, the Americas and more recently there have been a number of studies indicating a complex role for ENSO in affecting European climates albeit interacting with the Artic and North Atlantic Oscillation (e.g. Zanchettin et al., 2006). It should therefore be considered that changes in flood frequency in regional climates, whether historically or into the future, can occur as a function of entirely natural climate variability. It is clear that a more sophisticated approach to climate change detection for flood risk is required whereby causal physics are employed to aid the evaluation of changes in the climatic regime.

Acknowledgements. This paper represents an overview of a number of studies that have been funded by the Australian Research Council, Hunter Water Corporation and Sydney Catchment Authority.

\section{References}

Allan, R. J., Lindesay, J. A., and Reason, C. J. C.: Multi-decadal variability in the climate system over the Indian Ocean region during the austral summer, J. Climate, 8, 1853-1873, 1995.

Cornish, P. M: Changes in seasonal and annual rainfall in New South Wales, Search, 8, 38-40, 1977.

Erskine, W. D. and Warner, R. F.: "Geomorphic Effects of Alternating Flood and Drought Dominated Regimes on a NSW Coastal River" in: Fluvial Geomorphology of Australia, edited by: Warner, R. F., Academic Press, Sydney, Australia, 223-244, 1988.

Franks, S. W.: Identification of a change in climate state using regional flood data, Hydrol. Earth Syst. Sci., 6, 11-16, doi:10.5194/hess-6-11-2002, 2002.

Franks, S. W. and Kuczera, G.: Flood frequency analysis: evidence and implications of secular climate variability, Water Resour. Res., 38, 1062, doi:10.1029/2001WR000232, 2002.

Kiem, A. S., Franks, S. W., and Kuczera, G.: Multi-decadal variability of flood risk, Geophys. Res. Lett., 30, 1035, doi:10.1029/2002GL015992, 2003.

Kundzewicz, Z. W. and Robson, A. J.: Change detection in river flow records - review of methodology, Hydrol. Sci. J., 49, 7-19, 2004.

Micevski, T., Franks S. W., Kuczera, G.: Multidecadal variability in coastal eastern Australian flood data, J. Hydrol., 327, 219-225, 2006. 
Verdon, D. C. and Franks, S. W.: Long-term behaviour of ENSO: interactions with the PDO over the past 400 years inferred from paleoclimate records, Geophys. Res. Lett., 33, L06712, doi:10.1029/2005GL025052, 2006.
Zanchettin, D., Franks, S. W., Traverso, P., and Tomasino, M.: On ENSO impacts on European wintertime rainfalls and their modulation by the NAO and the Pacific multi-decadal variability described through the PDO index, Int. J. Climatol., 28, 995-1006, 2008. 